\begin{tabular}{|c|c|}
\hline Title & Finite element modeling of broad-band traveling-wave optical modul ators \\
\hline Author(s) & Koshiba, Masanori; Tsuji, Y asuhide; Nishio, Masahiro \\
\hline Citation & $\begin{array}{l}\text { IEEE TRANSA CTIONS ON MICROWA VE THEORY AND TECHNIQUES, 47(9), 1627-1633 } \\
\text { https://doi.org/10.1109/22.788602 }\end{array}$ \\
\hline Issue Date & 1999-09 \\
\hline Doc URL & http:/hdl. handle.net/2115/5586 \\
\hline Rights & $\begin{array}{l}\text { O1999 IEEE. Personal use of this material is permitted. However, permission to reprint/republish this material for } \\
\text { advertising or promotional purposes or for creating new collective works for resale or redistribution to servers or lists, } \\
\text { or to reuse any copyrighted component of this work in other works must be obtained from the IEEE." }\end{array}$ \\
\hline Type & article \\
\hline File Information & ITMTT 47-9.pdf \\
\hline
\end{tabular}

Instructions for use 


\title{
Finite-Element Modeling of Broad-Band Traveling-Wave Optical Modulators
}

\author{
Masanori Koshiba, Senior Member, IEEE, Yasuhide Tsuji, Member, IEEE, and Masahiro Nishio
}

\begin{abstract}
A full-wave finite-element method with hybrid edge/ nodal elements is, for the first time, applied to investigating the frequency dispersion of microwave propagation characteristics of broad-band traveling-wave (TW) optical modulators using planar electrode configurations. In order to produce a two-step analysis of electrooptic modulation of optical waveguides, the microwave electrode solver is linked to the optical waveguide solver. Numerical results are shown for an ultrabroad-band TW $\mathrm{LiNbO}_{3}$ Mach-Zehnder optical modulator with a ridge structure, and the necessity of using the full-wave solver is verified by comparing the calculated 3-dB bandwidth and half-wavelength voltage with the experimental data.
\end{abstract}

Index Terms - Coplanar waveguide, finite-element method, fullwave analysis, microwave photonic device, traveling-wave optical modulator.

\section{INTRODUCTION}

A BROAD-BAND optical modulator with a traveling-wave (TW) electrode is essential for future optical communication systems. Design of high-performance TW optical modulators using planar electrode configurations such as coplanar waveguide (CPW) or microstrip (MS) electrodes demands precise modeling and control of microwave propagation characteristics. For this purpose, various numerical techniques have been developed. In particular, the finite-element method (FEM) is a powerful and efficient tool for the most general waveguiding problems and has been widely used for modeling and optimization of the TW electrode [1]-[6]. The conventional FEM approach, however, is based on a quasiTEM approximation and, thus, the frequency dispersion of a microwave effective index, characteristic impedance, and attenuation constant, which are key parameters for realizing high-speed optical modulators, cannot be evaluated.

In this paper, the full-wave FEM is, for the first time, applied to investigating the frequency dispersion of microwave propagation characteristics of broad-band TW optical modulators. In order to eliminate spurious solutions, hybrid edge/nodal elements are introduced. The conventional FEM using the quasi-TEM approximation for the microwave analysis and the scalar FEM using the quasi-TE or quasi-TM approximation for the optical waveguide analysis are also briefly reviewed. When considering the push-pull electrooptic effect of a Mach-Zehnder-type modulator with parallel optical waveguides, the half-wavelength voltage is, in general, calculated by using the overlap integral factors between the

Manuscript received January 23, 1998.

The authors are with the Division of Electronics and Information Engineering, Hokkaido University, Sapporo 060-8628, Japan.

Publisher Item Identifier S 0018-9480(99)06586-2.

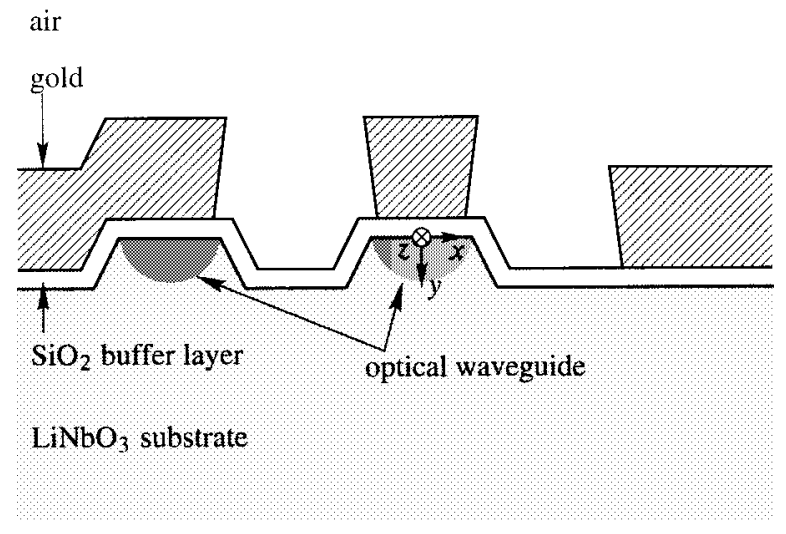

Fig. 1. TW optical modulator.

microwave electric field and optical power distribution for each electrooptically unmodulated optical waveguide. In this approach, on the other hand, to produce a two-step analysis of electrooptic modulation of optical waveguides, the fullwave and quasi-TEM FEM microwave solvers are linked to the scalar FEM optical waveguide solver and, therefore, more precise evaluation of the half-wavelength voltage using the optical effective index changes due to the microwave electric field in an optical waveguide is possible. Using these FEM solvers, the behavior of an ultrabroad-band $\mathrm{TW} \mathrm{LiNbO}_{3}$ Mach-Zehnder optical modulator with a ridge structure [7], [8] is investigated, and the necessity of using the full-wave FEM solver is verified by comparing the numerical results with the experimental data [7], [8].

\section{OpticAl ReSPONSE AND HALF-WAVELENGTH VoltagE}

We consider a Mach-Zehnder-type modulator with a gold CPW electrode, as shown in Fig. 1 [6]-[8], as a typical TW optical modulator. A Ti-diffused optical waveguide is formed in a $Z$-cut and $X$-propagation $\mathrm{LiNbO}_{3}$ substrate with a ridge structure. The CPW structure incorporates finite electrode thickness and conductivity, and metallization undercutting resulting from various metallization processes.

The optical response $m(f)$ is given by [9]

$$
\begin{aligned}
m(f)= & \mid \frac{1-S_{1} S_{2}}{\left(1+S_{2}\right)\left[\exp \left(j 2 u_{+}\right)-S_{1} S_{2} \exp \left(-j 2 u_{-}\right)\right]} \\
& \quad \times\left[\exp \left(j u_{+}\right) \frac{\sin u_{+}}{u_{+}}+S_{2} \exp \left(-j u_{-}\right) \frac{\sin u_{-}}{u_{-}}\right] \mid
\end{aligned}
$$


with

$$
\begin{aligned}
S_{1} & =\frac{Z_{1}-Z}{Z_{1}+Z} \\
S_{2} & =\frac{Z_{2}-Z}{Z_{2}+Z} \\
u_{ \pm} & =\frac{1}{c} \pi f l\left(n_{m} \mp n_{\mathrm{eff}}\right)-j \frac{1}{2} \alpha l
\end{aligned}
$$

where $Z, Z_{1}$, and $Z_{2}$ are the microwave characteristic impedance, the microwave generator internal impedance, and the shunted loaded impedance, respectively, $f$ is the microwave frequency, $n_{m}$ is the microwave effective index, $\alpha$ is the microwave attenuation constant, $n_{\text {eff }}$ is the optical effective index, $c$ is the light velocity of free space, and $l$ is the electrode interaction length. To realize broad-band optical modulators, the velocity-matching condition $n_{m}=n_{\mathrm{eff}}$ and the impedance-matching condition $Z=Z_{1}=Z_{2}$ should be achieved, and the microwave propagation loss should also be reduced.

When considering the push-pull electrooptic effect of a Mach-Zehnder-type modulator with parallel optical waveguides 1 and 2, the half-wavelength voltage $V_{\pi}$ is determined via the relation

$$
\left(\left|\Delta n_{\mathrm{eff} 1}\right|+\left|\Delta n_{\mathrm{eff} 2}\right|\right) l=\frac{\lambda}{2}
$$

where $\lambda$ is the free-space optical wavelength, and $\Delta n_{\text {eff } 1}$ and $\Delta n_{\text {eff2 }}$ are the optical effective index changes due to the microwave electric field in optical waveguides 1 and 2 , respectively. The half-wavelength voltage can also be approximated as

$$
V_{\pi} l=\frac{\lambda}{\left|\Gamma_{1}\right|+\left|\Gamma_{2}\right|}
$$

where $\Gamma_{1}$ and $\Gamma_{2}$ are the overlap integral factors between the microwave electric field and the optical power distribution for optical waveguides 1 and 2, respectively. In the conventional design of TW optical modulators, the half-wavelength voltage has been evaluated via (6) because, in general, it is very cumbersome to analyze the modified waveguide with electrooptic induced changes in the refractive index. In this approach, to produce a two-step analysis of electrooptic modulation of optical waveguides, the microwave solver is linked to the optical waveguide solver and, thus, more precise evaluation of the half-wavelength voltage using (5) is also possible.

\section{MicrowaVe ANALYsis}

\section{A. Full-Wave Analysis}

To evaluate the microwave frequency dispersion of a CPW electrode, shown in Fig. 1, the full-wave analysis is necessary, and different types of full-wave FEM solvers have been developed. Of the various solvers, the FEM in terms of the electric or magnetic field vector $\boldsymbol{E}$ or $\boldsymbol{H}$ with hybrid edge/nodal triangular elements [10]-[12] is quite suitable for arbitrarily-shaped TW electrodes, where the edge and nodal elements are used for the transverse and axial fields, respectively.

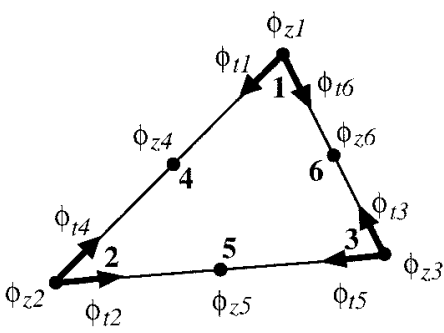

Fig. 2. High-order hybrid edge/nodal triangular element.

From Maxwell's equations, the vector wave equation is derived as

$$
\nabla \times \nabla \times \boldsymbol{E}-k_{0}^{2}\left[\varepsilon_{r}\right] \boldsymbol{E}=0
$$

where $k_{0}$ is the free-space wavenumber, and $\left[\varepsilon_{r}\right]$ is the diagonal relative permittivity tensor. We use the electric field as the working variable because it is directly related with the current flowing along the CPW electrode with finite conductivity.

Fig. 2 shows the high-order hybrid edge/nodal triangular element [12], which is composed of a linear edge element with six tangential unknowns at the three vertices of the triangle, $E_{t 1}$ to $E_{t 6}$, and a quadratic nodal element with six axial unknowns, $E_{z 1}$ to $E_{z 6}$. The electric field within each element is expressed as

$$
\boldsymbol{E}=\left[\begin{array}{c}
E_{x} \\
E_{y} \\
E_{z}
\end{array}\right]=\left[\begin{array}{c}
\{U\}^{T}\left\{E_{t}\right\}_{e} \\
\{V\}^{T}\left\{E_{t}\right\}_{e} \\
j \beta\{N\}^{T}\left\{E_{z}\right\}_{e}
\end{array}\right]
$$

where the components of the vectors $\left\{E_{t}\right\}_{e}$ and $\left\{E_{z}\right\}_{e}$ are $E_{t 1}$ to $E_{t 6}$ and $E_{z 1}$ to $E_{z 6}$, respectively, $\{U\}$ and $\{V\}$ are the shape function vectors for the linear edge triangular element, $\{N\}$ is the shape function vector for the quadratic nodal triangular element, $T$ denotes a transpose, and $\beta$ is the phase constant in the $z$-direction. In a lossy case, $\beta$ is replaced by $\beta-j \alpha$.

Application of the FEM procedure to (7) gives the following matrix equation:

$$
[K]\{E\}=\beta^{2}[M]\{E\}
$$

with

$$
\begin{aligned}
& {[K]=\left[\begin{array}{cc}
{\left[K_{t t}\right]} & {[0]} \\
{[0]} & {[0]}
\end{array}\right]} \\
& {[M]=\left[\begin{array}{ll}
{\left[M_{t t}\right]} & {\left[M_{t z}\right]} \\
{\left[M_{z t}\right]} & {\left[M_{z z}\right]}
\end{array}\right]}
\end{aligned}
$$

where the components of the vector $\{E\}$ are composed of all edge and nodal variables, $[0]$ is a null matrix, and the submatrices of global matrices $[K]$ and $[M]$ are expressed as

$$
\begin{aligned}
{\left[K_{t t}\right] } & =\sum_{e} \iint_{e}\left[k_{0}^{2}\left(\varepsilon_{r x}\{U\}\{U\}^{T}+\varepsilon_{r y}\{V\}\{V\}^{T}\right)\right. \\
& \left.-\left(\frac{\partial\{V\}}{\partial x}-\frac{\partial\{U\}}{\partial y}\right) \times\left(\frac{\partial\{V\}^{T}}{\partial x}-\frac{\partial\{U\}^{T}}{\partial y}\right)\right] \\
& \times d x d y
\end{aligned}
$$




$$
\begin{aligned}
& {\left[M_{t t}\right]} \\
& =\sum_{e} \iint_{e}\left(\{U\}\{U\}^{T}+\{V\}\{V\}^{T}\right) d x d y \\
& {\left[M_{t z}\right]} \\
& =\sum_{e} \iint_{e}\left(\{U\} \frac{\partial\{N\}^{T}}{\partial x}+\{V\} \frac{\partial\{N\}^{T}}{\partial y}\right) d x d y \\
& =\left[M_{z t}\right]^{T} \\
& {\left[M_{z z}\right]} \\
& =\sum_{e} \iint_{e}\left(\frac{\partial\{N\}}{\partial x} \frac{\partial\{N\}^{T}}{\partial x}+\frac{\partial\{N\}}{\partial y} \frac{\partial\{N\}^{T}}{\partial y}\right. \\
& \left.\quad-k_{0}^{2} \varepsilon_{r z}\{N\}\{N\}^{T}\right) d x d y .
\end{aligned}
$$

Here, $\varepsilon_{r x}, \varepsilon_{r y}$, and $\varepsilon_{r z}$ are the relative permittivities in the $x-, y-$ and $z$-directions, respectively, and the summation $\sum_{e}$ extends over all different elements.

Noting that the band of the sparse matrices $[K]$ and $[M]$ can be reduced by reordering the components of $\{E\}$, the largescale complex generalized eigenvalue equation (9) is solved to give the phase and attenuation constants as the eigenvalues by using the subspace iteration method [13], and the microwave effective index is then given by $n_{m}=\beta / k_{0}$. The characteristic impedance of the $\mathrm{CPW}$ electrode is calculated using the power-current definition

$$
Z_{c}=\frac{2 P}{|I|^{2}}
$$

where $P$ is the modal power, and $I$ is the total $z$-directional current carried by the center electrode. They are related to the field quantities corresponding to the eigenvectors of (9) as

$$
\begin{aligned}
P & =\frac{1}{2} \iint\left(\boldsymbol{E} \times \boldsymbol{H}^{*}\right) \cdot \boldsymbol{i}_{z} d x d y \\
& =\frac{\beta^{*}}{\omega \mu_{0}}\left(\left\{E_{t}\right\}^{T}\left[M_{t t}\right]\left\{E_{t}\right\}^{*}+\left\{E_{t}\right\}^{T}\left[M_{t z}\right]\left\{E_{z}\right\}^{*}\right) \\
I & =\iint \sigma E_{z} d x d y \\
& =\sum_{e}^{\prime} \iint_{e} j \beta \sigma\{N\}^{T}\left\{E_{z}\right\}_{e} d x d y
\end{aligned}
$$

where the integration is done over the entire waveguide cross section for $P$ and over the center electrode for $I, \omega$ is the angular frequency, $\mu_{0}$ is the permeability of free space, $\sigma$ is the electrode conductivity, $\boldsymbol{i}_{z}$ is the unit vector in the $z$-direction, the asterisk denotes a complex conjugate, and the summation $\sum_{e}^{\prime}$ extends over the elements on the center electrode.

\section{B. Quasi-TEM Analysis}

When the quasi-TEM wave propagation is assumed, the electric-field calculation is reduced to an electrostatic-field problem governed by Laplace's equation. The TEM matrix equation for Laplace's equation is given by

$$
[K]\{V\}=\{Q\}
$$

with

$$
\begin{aligned}
{[K]=\sum_{e} \iint_{e} \varepsilon_{0}\left(\varepsilon_{r x} \frac{\partial\{N\}}{\partial x} \frac{\partial\{N\}^{T}}{\partial x}\right.} & \\
& \left.+\varepsilon_{r y} \frac{\partial\{N\}}{\partial y} \frac{\partial\{N\}^{T}}{\partial y}\right) d x d y
\end{aligned}
$$

where the quadratic nodal triangular elements are used for discretizing the waveguide cross section, except electrode regions, $\{V\}$ is the nodal electrostatic potential vector, the vector $\{Q\}$ originates from imposed voltages and corresponds to the surface charge density distribution on the electrode, and $\varepsilon_{0}$ is the permittivity of free space.

From the nodal potential values, the capacitance per unit length of the CPW electrode is evaluated as

$$
C=\frac{1}{V_{0}^{2}}\{V\}^{T}[K]\{V\}
$$

where $V_{0}$ is the applied voltage. The microwave effective index and characteristic impedance are given by

$$
\begin{aligned}
n_{m} & =\sqrt{\frac{C}{C_{0}}} \\
Z_{c} & =\frac{1}{c \sqrt{C C_{0}}}
\end{aligned}
$$

where $C_{0}$ is the free-space capacitance per unit length of the CPW electrode. The attenuation constant due to the conductor loss is calculated by using the incremental inductance formula [14]

$$
\alpha=\frac{R_{s}}{2 \mu_{0} Z_{c}} \frac{\partial L}{\partial n}=\frac{R_{s}}{2 Z_{0} Z_{c}} \frac{\partial Z_{c 0}}{\partial n}
$$

where $L$ is the inductance per unit length and remains unaltered for nonmagnetic materials, $R_{S}$ is the surface resistance of the conductor, $Z_{0}=\sqrt{\mu_{0} / \varepsilon_{0}}$ is the impedance of free space, $Z_{c 0}$ is the free-space characteristic impedance of the $\mathrm{CPW}$ electrode, and $\partial Z_{c 0} / \partial n$ denotes the derivative of $Z_{c 0}$ with respect to the incremental recession of electrode surfaces.

\section{Optical WaVEguide ANALYsis}

Although a formulation based on a single scalar quantity is inadequate for the arbitrarily shaped waveguides with an arbitrary transverse distribution of permittivity or refractive index, useful approximations can be found in the form of quasi-TE or quasi-TM modes, depending on the type of waveguiding structure or the propagation mode of interest [15], [16]. These approximations can be sufficiently accurate for many practical cases of Ti: $\mathrm{LiNbO}_{3}$ optical waveguides [17], [18].

From Maxwell's equations, the scalar wave equation is derived as

$$
\frac{n_{x}^{2}}{n_{z}^{2}} \frac{\partial^{2} E_{x}}{\partial x^{2}}+\frac{\partial^{2} E_{x}}{\partial y^{2}}-\beta^{2} E_{x}+k_{0}^{2} n_{x}^{2} E_{x}=0
$$

for quasi-TE modes and

$$
\frac{1}{n_{y}^{2}} \frac{\partial^{2} H_{x}}{\partial x^{2}}+\frac{1}{n_{z}^{2}} \frac{\partial^{2} H_{x}}{\partial y^{2}}-\frac{\beta^{2}}{n_{y}^{2}} H_{x}+k_{0}^{2} H_{x}=0
$$


for quasi-TM modes, where $n_{x}, n_{y}$, and $n_{z}$ are the refractive indexes in the $x$-, $y$-, and $z$-directions, respectively.

Application of the FEM procedure using the quadratic nodal triangular elements to (25) and (26) gives the following matrix eigenvalue equations:

$$
[K]\left\{E_{x}\right\}=\beta^{2}[M]\left\{E_{x}\right\}
$$

with

$$
\begin{gathered}
{[K]=\sum_{e} \iint_{e}\left(k_{0}^{2} n_{x}^{2}\{N\}\{N\}^{T}-\frac{n_{x}^{2}}{n_{z}^{2}} \frac{\partial\{N\}}{\partial x} \frac{\partial\{N\}^{T}}{\partial x}\right.} \\
\left.-\frac{\partial\{N\}}{\partial y} \frac{\partial\{N\}^{T}}{\partial y}\right) d x d y \\
{[M]=\sum_{e} \iint_{e}\{N\}\{N\}^{T} d x d y}
\end{gathered}
$$

for quasi-TE modes, and

$$
[K]\left\{H_{x}\right\}=\beta^{2}[M]\left\{H_{x}\right\}
$$

with

$$
\begin{gathered}
{[K]=\sum_{e} \iint_{e}\left(k_{0}^{2}\{N\}\{N\}^{T}-\frac{1}{n_{y}^{2}} \frac{\partial\{N\}}{\partial x} \frac{\partial\{N\}^{T}}{\partial x}\right.} \\
\left.-\frac{1}{n_{z}^{2}} \frac{\partial\{N\}}{\partial y} \frac{\partial\{N\}^{T}}{\partial y}\right) d x d y \\
{[M]=\sum_{e} \iint_{e} \frac{1}{n_{y}^{2}}\{N\}\{N\}^{T} d x d y}
\end{gathered}
$$

for quasi-TM modes. The optical effective index is given by $n_{\mathrm{eff}}=\beta / k_{0}$.

\section{Numerical Results}

We consider a $\mathrm{Ti}: \mathrm{LiNbO}_{3}$ Mach-Zehnder optical modulator with a gold CPW electrode in Fig. 1.

In the microwave frequency range, the relative permittivities of the $Z$-cut $\mathrm{LiNbO}_{3}$ substrate are 28 and 43 perpendicular and parallel to the substrate surface, respectively, the relative permittivity of the $\mathrm{SiO}_{2}$ buffer layer is 3.9 , and the conductivity of the gold electrode is $4.1 \times 10^{7} \mathrm{~S} / \mathrm{m}$.

In the optical frequency range, the ordinary and extraordinary refractive index profiles of the $\mathrm{Ti}: \mathrm{LiNbO}_{3}$ optical waveguide $n_{o}$ and $n_{e}$ are given by [17], [18]

$$
\begin{aligned}
& n_{o}=n_{o s}+\Delta n_{s}[f(x) g(y)]^{0.5 \tilde{5}} \\
& n_{e}=n_{e s}+\Delta n_{e} f(x) g(y)
\end{aligned}
$$

with

$$
\begin{aligned}
& f(x)=\frac{\operatorname{erf}\left(\frac{2 x+W}{2 d_{x}}\right)-\operatorname{erf}\left(\frac{2 x-W}{2 d_{x}}\right)}{2 \operatorname{erf}\left(\frac{W}{2 d_{x}}\right)} \\
& g(y)=\exp \left[-\left(\frac{y}{d_{y}}\right)^{2}\right]
\end{aligned}
$$

where $n_{o s}$ and $n_{e s}$ are the ordinary and extraordinary refractive indexes of $\mathrm{LiNbO}_{3}$, respectively, $\Delta n_{o}$ and $\Delta n_{s}$ are the

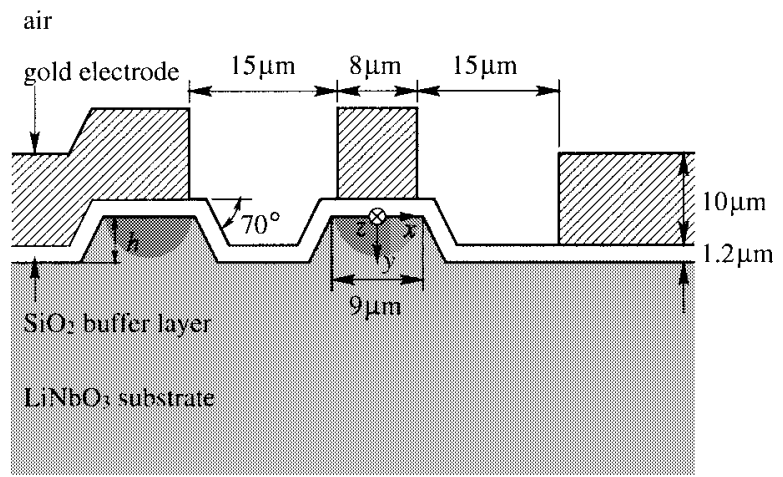

Fig. 3. Rectangular CPW electrode.

maximum ordinary and extraordinary refractive index changes, respectively, $W$ is the Ti stripe width before indiffusion, and $d_{x}$ and $d_{y}$ are the diffusion lengths in the $x$ - and $y$ directions, respectively, The profile function of (35) is used for the right optical waveguide in Fig. 1. For the left one, $x$ is replaced by $x-s$ with $s$ being the waveguide separation. According to [7], [17], and [18], the operating wavelength is assumed to be $\lambda=1.55 \mu \mathrm{m}$, and $n_{s o}=2.214, n_{s e}=2.138$, $\Delta n_{o}=0.0062, \Delta n_{e}=0.0146, W=6 \mu \mathrm{m}, d_{x}=4.850 \mu \mathrm{m}$, and $d_{y}=4.105 \mu \mathrm{m}$. The refractive indexes of the $Z$-cut $\mathrm{LiNbO}_{3}$ substrate are given by $n_{x}=n_{z}=n_{o}$ and $n_{y}=n_{e}$, and the refractive indexes of the $\mathrm{SiO}_{2}$ buffer layer and gold electrode are 1.45 and $0.379-j 10.75$, respectively.

The refractive index changes due to the electrooptic effect are calculated as

$$
\begin{aligned}
\Delta n_{x} & =-\frac{n_{x}^{3}}{2}\left(\gamma_{22} E_{m x}+\gamma_{13} E_{m y}\right) \\
\Delta n_{y} & =-\frac{n_{y}^{3}}{2} \gamma_{33} E_{m y} \\
\Delta n_{z} & =-\frac{n_{z}^{3}}{2}\left(-\gamma_{22} E_{m x}+\gamma_{13} E_{m y}\right)
\end{aligned}
$$

for the $Z$-cut and $X$-propagation $\mathrm{LiNbO}_{3}$ substrate, where $E_{m x}$ and $E_{m y}$ are the microwave electric fields, and the electrooptic coefficients are $\gamma_{22}=3.4 \times 10^{-12} \mathrm{~m} / \mathrm{V}, \gamma_{13}=$ $8.6 \times 10^{-12} \mathrm{~m} / \mathrm{V}$, and $\gamma_{33}=30.8 \times 10^{-12} \mathrm{~m} / \mathrm{V}$. To exploit the dominant $\mathrm{LiNbO}_{3}$ electrooptic coefficient $\gamma_{33}$, the quasi-TM optical mode propagation is chosen.

We first consider a rectangular $\mathrm{CPW}$ electrode with no metallization undercutting, as shown in Fig. 3 [7]. Fig. 4(a) shows the frequency dispersion of the microwave effective index $n_{m}$ and attenuation constant $\alpha$ and Fig. 4(b) shows that of real and imaginary parts of the characteristic impedance, $\operatorname{Re}\left[Z_{c}\right]$ and $\operatorname{Im}\left[Z_{c}\right]$, where the ridge height is assumed to be $h=3.3 \mu \mathrm{m}$. In contrast with the quasi-TEM analysis, the full-wave analysis shows that the microwave effective index can vary appreciably with frequencies. In the current full-wave analysis, the field penetration into the electrode is taken into account. Fig. 5 shows the magnitude of electric-field vector $|\boldsymbol{E}|$ in the center electrode. The corner frequency [19] at which the ratio of electrode thickness to skin depth equals three is $f_{c}=0.56 \mathrm{GHz}$ for the gold electrode with a thickness of $10 \mu \mathrm{m}$. The familiar $\sqrt{f}$ dependence of $\alpha$ with $f$ being the microwave frequency holds only for $f>f_{c}$. The finite 


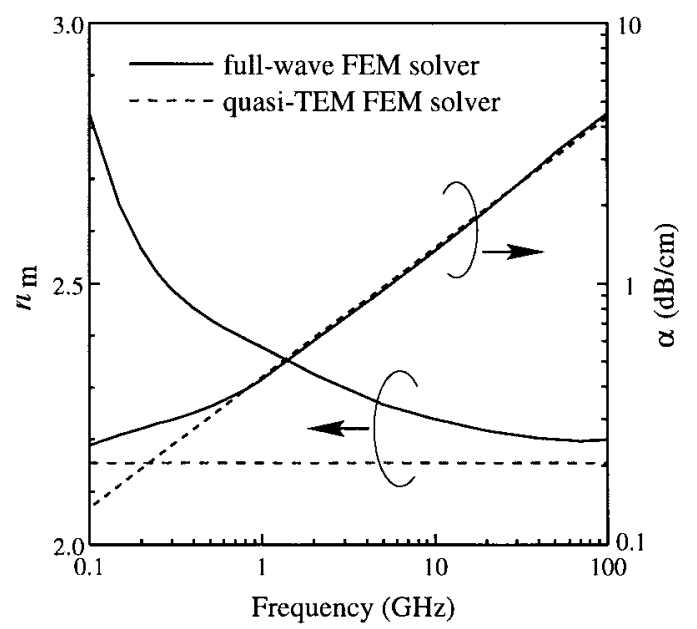

(a)

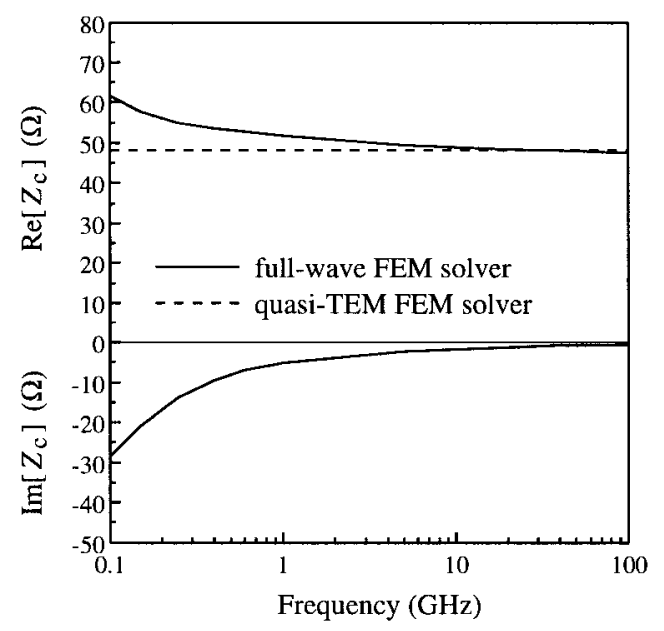

(b)

Fig. 4. Microwave propagation characteristics of a rectangular CPW electrode. (a) Microwave effective index and attenuation constant. (b) Characteristic impedance.

electrode conductivity also severely affects the characteristic impedance that should have a negative imaginary part. The increases of $n_{m}, \operatorname{Re}\left[Z_{c}\right]$, and $\operatorname{Im}\left[Z_{c}\right]$ at low frequencies are attributed to the simultaneous increases in $L$ and $R /(\omega L)$ [19], where $R$ and $L$ are the equivalent line resistance and inductance, respectively. Fig. 6 shows the optical response, where $Z_{1}=Z_{2}=50 \Omega, l=2 \mathrm{~cm}$, and the optical effective index of the fundamental quasi-TM mode propagating in the electrooptically unmodulated $\mathrm{Ti}: \mathrm{LiNbO}_{3}$ waveguide is $n_{\mathrm{eff}}=$ 2.142. The optical response calculated by using the full-wave FEM solver is in good agreement with the experimental data [7]. In Fig. 6, the optical response of the conventional CPW electrode on a flat substrate $(h=0)$ is also presented.

We next consider a trapezoidal CPW electrode with metallization undercutting, as shown in Fig. 7 [8]. Fig. 8(a) shows the frequency dispersion of the microwave effective index and attenuation constant and Fig. 8(b) shows that of real and imaginary parts of the characteristic impedance. As noted in [20], the microwave propagation characteristics of a thick CPW electrode depend on the sidewall inclination. The fullwave analysis shows that the microwave effective index and

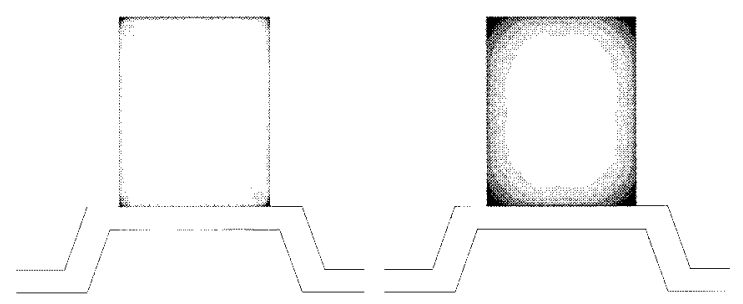

(a)

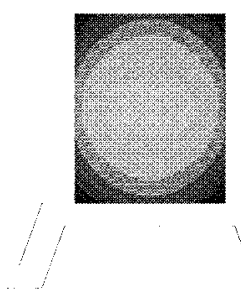

(c) (b)

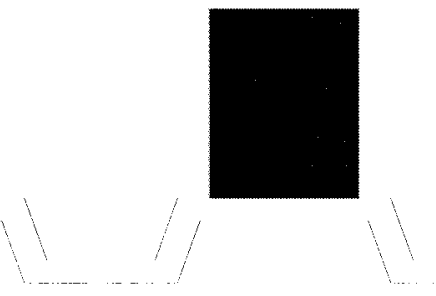

(d)
Fig. 5. Electric-field penetration into a center electrode at: (a) $f=100 \mathrm{GHz}$, (b) $f=10 \mathrm{GHz}$, (c) $f=1 \mathrm{GHz}$, and (d) $f=0.1 \mathrm{GHz}$.

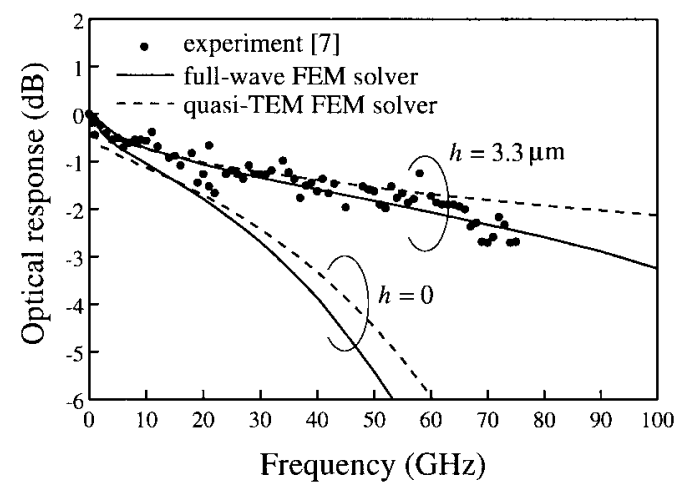

Fig. 6. Optical response of a rectangular CPW electrode.

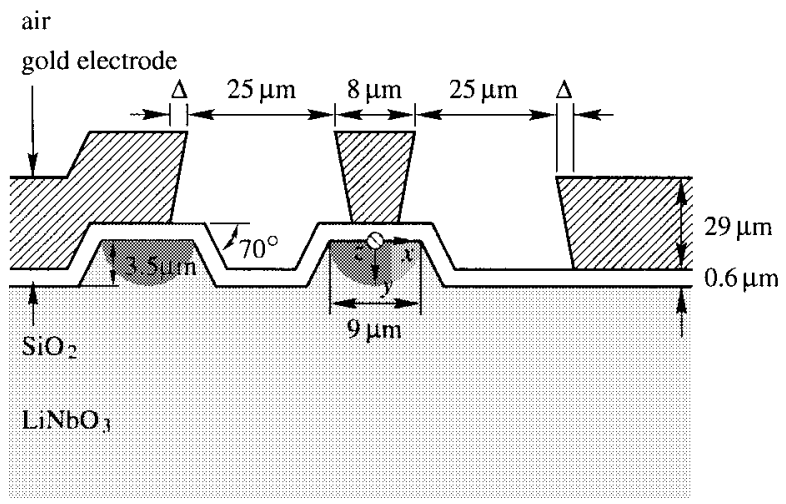

Fig. 7. Trapezoidal CPW electrode.

the real part of characteristic impedance are strongly dependent on the electrode sidewall inclination. Fig. 9 shows the optical response, where the values of $Z_{1}, Z_{2}, l$, and $n_{\mathrm{eff}}$ are the same as those in Fig. 6. The optical response of the trapezoidal CPW electrode with metallization undercutting of $\Delta=1 \mu \mathrm{m}$ is in good agreement with the experimental data [8]. Fig. 10 shows the product of half-wavelength voltage and electrode interaction length $V_{\pi} l$, which is calculated by using (5). It is confirmed that the metallization undercutting increases the 


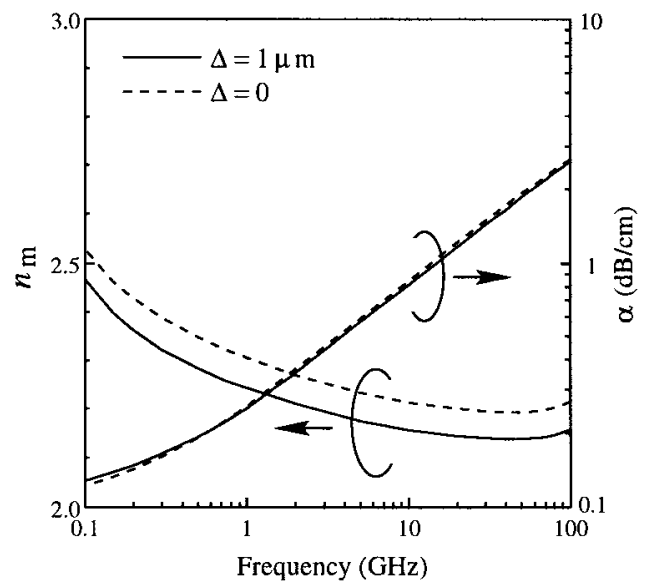

(a)

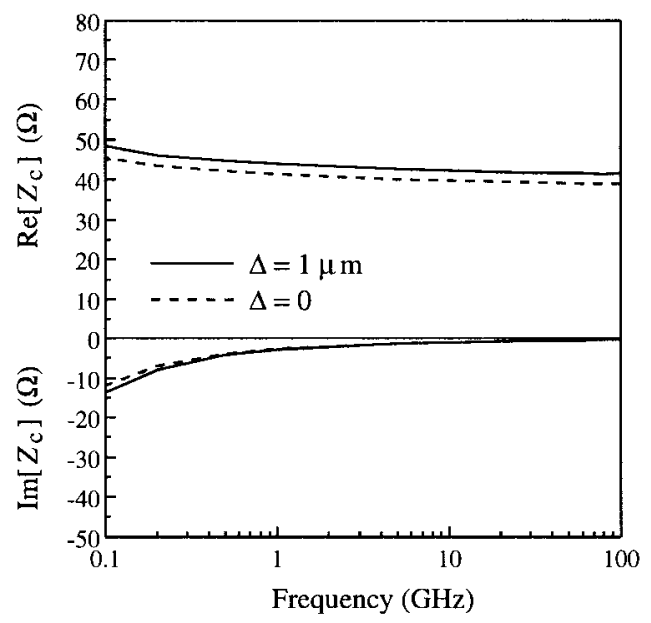

(b)

Fig. 8. Microwave propagation characteristics of a trapezoidal CPW electrode. (a) Microwave effective index and attenuation constant. (b) Characteristic impedance.

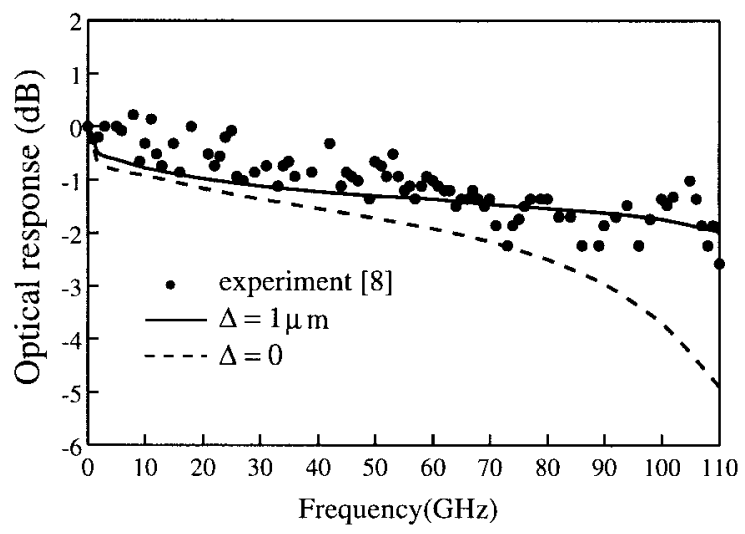

Fig. 9. Optical response of a trapezoidal CPW electrode.

half-wavelength voltage. The value of $V_{\pi} l$ is also evaluated via (6), where the overlap integral factor is given by

$$
\Gamma_{i}=\frac{\iint \gamma_{33} n_{e}^{3} E_{m y}(x, y) I_{0}(x, y) d x d y}{V_{0} \iint I_{0}(x, y) d x d y}
$$

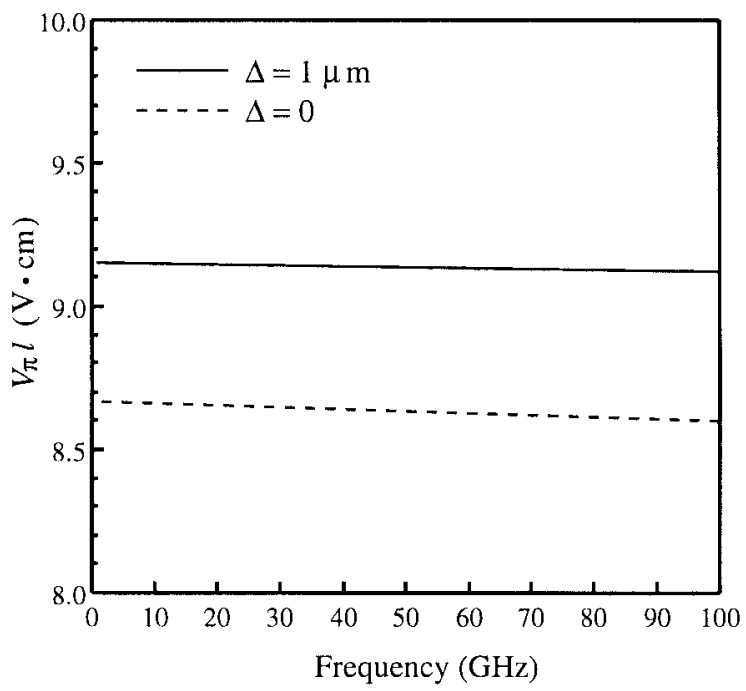

Fig. 10. Product of half-wavelength voltage and electrode interaction length.

with $I_{0}=\operatorname{Re}\left[\left|H_{x}\right|^{2} / n_{y}^{2}\right]$ being the optical power distribution. In the structure considered here, the results of (6) are approximately with those of (5).

\section{CONCLUSION}

A full-wave FEM with hybrid edge/nodal elements was applied to investigating the frequency dispersion of microwave propagation characteristics of broad-band TW optical modulators with planar electrode structures. In order to produce a two-step analysis of electrooptic modulation of optical waveguides, the microwave electrode solver was linked to the optical waveguide solver. Numerical results were shown for an ultrabroad-band TW $\mathrm{LiNbO}_{3}$ Mach-Zehnder optical modulator with a ridge structure. An FEM-based modeling of TW photodetectors is currently under consideration.

\section{REFERENCES}

[1] J. C. Yi, S. H. Kim, and S. S. Choi, "Finite-element method for the impedance analysis of traveling-wave modulators," J. Lightwave Technol., vol. 8, pp. 817-822, June 1990.

[2] K. Kawano, K. Noguchi, T. Kitoh, and H. Miyazawa, "A finite-element method (FEM) analysis of a shielded velocity-matched $\mathrm{Ti}: \mathrm{LiNbO}_{3}$ optical modulator," IEEE Photon. Technol. Lett., vol. 3, pp. 919-921, Oct. 1991.

[3] K. Kawano, "Improvement of the performance of a shielded velocitymatched $\mathrm{Ti}: \mathrm{LiNbO}_{3}$ optical modulator," IEICE Trans. Electron., vol. E76-C, pp. 238-243, Feb. 1993.

[4] G. K. Gopalakrishnan, W. K. Burns, R. W. McElhanon, C. H. Bulmer, and A. S. Greenblatt, "Performance and modeling of broad-band $\mathrm{LiNbO}_{3}$ traveling-wave optical intensity modulators," J. Lightwave Technol., vol. 12, pp. 1807-1819, Oct. 1994.

[5] X. Zhang and T. Miyoshi, "Optimum design of coplanar waveguide for $\mathrm{LiNbO}_{3}$ optical modulator," IEEE Trans. Microwave Theory Tech., vol. 43, pp. 523-528, Mar. 1995.

[6] O. Mitomi, K. Noguchi, and H. Miyazawa, "Design of ultra-broadband $\mathrm{LiNbO}_{3}$ optical modulators with ridge structure," IEEE Trans. Microwave Theory Tech., vol. 43, pp. 2203-2207, Sept. 1995.

[7] K. Noguchi, O. Mitomi, H. Miyazawa, and S. Seki, "A broad-band $\mathrm{Ti}: \mathrm{LiNbO}_{3}$ optical modulator with a ridge structure," J. Lightwave Technol., vol. 13, pp. 1164-1168, June 1995.

[8] K. Noguchi, O. Mitomi, and H. Miyazawa, "Low-voltage and broadband $\mathrm{Ti}: \mathrm{LiNbO}_{3}$ modulators operating in the millimeter wavelength region," in Opt. Fiber Commun. Tech. Dig., Feb./Mar. 1997, pp. 205-206.

[9] S. Uehara, "Focusing-type optical modulator," IEEE J. Quantum Electron., vol. QE-9, pp. 984-986, Oct. 1973. 
[10] J.-F. Lee, D.-K. Sun, and Z. J. Cendes, "Full-wave analysis of dielectric waveguides using tangential vector finite elements," IEEE Trans. Microwave Theory Tech., vol. 39, pp. 1262-1271, Aug. 1991.

[11] M. Koshiba and K. Inoue, "Single and efficient finite-element analysis of microwave and optical waveguides," IEEE Trans. Microwave Theory Tech., vol. 40, pp. 371-377, Feb. 1992.

[12] M. Koshiba, S. Maruyama, and K. Hirayama, "A vector finite-element method with the high-order mixed-interpolation-type triangular elements for optical waveguiding problems," J. Lightwave Technol., vol. 12, pp. 495-502, Mar. 1994.

[13] F. A. Fernandez, J. B. Davies, S. Zhu, and Y. Lu, "Sparse matrix eigenvalue solver for finite element solution of dielectric waveguides,' Electron. Lett., vol. 27, pp. 1824-1826, Sept. 1991.

[14] H. A. Wheeler, "Formulas for the skin effect," Proc. IRE, vol. 30, pp. 412-414, Sept. 1942

[15] N. Mabaya, P. E. Lagasse, and P. Vandenbulcke, "Finite-element analysis of optical waveguides," IEEE Trans. Microwave Theory Tech., vol. MTT-29, pp. 600-605, June 1981.

[16] M. Koshiba, K. Hayata, and M. Suzuki, "Approximate scalar finiteelement analysis of anisotropic optical waveguides," Electron. Lett., vol. 18, pp. 411-413, May 1982.

[17] E. Strake, G. P. Bava, and I. Montrosset, "Guided modes of Ti : $\mathrm{LiNbO}_{3}$ channel waveguides: A novel quasi-analytical technique in comparison with the scalar finite-element method," J. Lightwave Technol., vol. 6, pp. 1126-1135, June 1988.

[18] L. Bersiner, U. Hempelmann, and E. Strake, "Numerical analysis of finite-element method and beam-propagation method results," J. Opt. Soc. Amer. B, Opt. Phys. vol. 8, pp. 422-433, Feb. 1991.

[19] W.-K. Wang, R. W. Smith, and P. J. Anthony, "Full-wave analysis of coplanar waveguides for $\mathrm{LiNbO}_{3}$ optical modulators by the modematching method considering nonideal conductors on etched buffer layers," J. Lightwave Technol., vol. 13, pp. 2250-2257, Nov. 1995.

[20] M. Seino, N. Mekada, T. Yamane, Y. Kubota, and M. Doi, "20GHz 3-dB bandwidth Ti: $\mathrm{LiNbO}_{3}$ Mach-Zehnder modulator," in Proc. European Opt. Commun. Conf., vol. 3, Sept. 1990, pp. 999-1002.

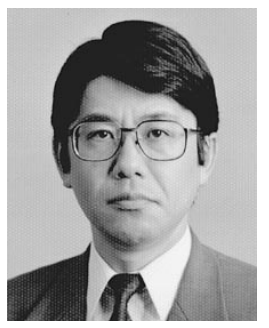

Masanori Kosiba (SM'84) was born in Sapporo, Japan, on November 23, 1948. He received the B.S., M.S., and Ph.D. degrees in electronic engineering from Hokkaido University, Sapporo, Japan, in 1971, 1973, and 1976, respectively.

In 1976, he joined the Department of Electronic Engineering, Kitami Institute of Technology, Kitami, Japan. From 1979 to 1987, he was an Associate Professor of electronic engineering at Hokkaido University and, in 1987, he became a Professor. He has been engaged in research on wave electronics, including microwaves, millimeter-waves, lightwaves, surface acoustic waves (SAW's), magnetostatic waves (MSW's), and electron waves, and computer-aided design and modeling of guided-wave devices using the FEM, boundary-element method, beam propagation method, etc. $\mathrm{He}$ has authored or co-authored over 200 research papers in English and over 100 research papers in Japanese, both in refereed journals. He authored Optical Waveguide Analysis (New York: McGraw-Hill, 1992) and Optical Waveguide Theory by the Finite Element Method (Norwell, MA: Kluwer, 1992), and co-authored Analysis Methods for Electromagnetic Wave Problems (Norwood, MA: Artech House, 1990), Ultrafast and Ultra-parallel Optoelectronics (New York: Wiley, 1995), and Finite Element Software for Microwave Engineering (New York: Wiley, 1996).

Dr. Koshiba is a member of the Institute of Electronics, Information and Communication Engineers (IEICE), Japan, the Institute of Electrical Engineers, Japan, the Institute of Image Information and Television Engineers, Japan, the Japan Society for Simulation Technology, the Japan Society for Computational Methods in Engineering, the Japan Society of Applied Electromagnetics and Mechanics, the Japan Society for Computational Engineering and Science, and the Applied Computational Electromagnetics Society (ACES). In 1987, 1997, and 1999, he was awarded the Excellent Paper Awards presented by the IEICE and, in 1998, he was awarded the Electronics Society Awards presented by the IEICE.

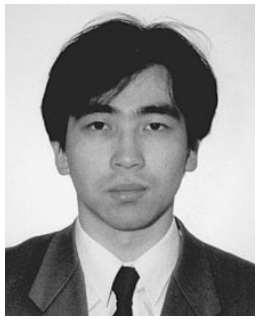

Yasuhide Tsuji (M'97) was born in Takikawa, Japan, on December 31, 1967. He received the B.S., M.S., and Ph.D. degrees in electronic engineering from Hokkaido University, Sapporo, Japan, in 1991, 1993, and 1996, respectively.

In 1996, he joined the Department of Applied Electronic Engineering, Hokkaido Institute of Technology and, since 1997, he has been an Associate Professor, engaged in research on wave electronics

Dr. Tsuji is a member of the Institute of Electronics, Information and Communication Engineers (IEICE), Japan. In 1997 and 1999, he was awarded the Excellent Paper Awards presented by the IEICE and, in 1999, he was awarded the Young Scientist Award presented by the IEICE.

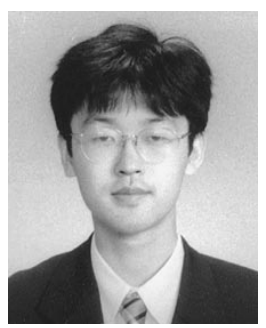

Masahiro Nishio was born in Otaru, Japan, on May 24, 1973. He received the B.S. degree in electronic engineering from Hokkaido University, Sapporo, Japan, in 1996, and is currently working at the university toward the M.S. degree in electronic and information engineering.

Mr. Nishio is a student member of the Institute of Electronics, Information and Communication Engineers (IEICE), Japan. 